\title{
Early Detection of Left Ventricular Systolic Dysfunction in Asymptomatic Patients with Chronic Aortic Regurgitation by two Dimensional Speckle Tracking Echocardiography
}

${ }^{1}$ Amal N. Al-Marayati, ${ }^{2}$ Thamer I. Al-Jawahiri

\begin{abstract}
Background: Early detection of subclinical left ventricular (LV) systolic dysfunction is crucial and could influence patients' prognosis by aiding the clinician to candidate patients for better management. Objective: To detect early LV systolic dysfunction in asymptomatic patient with chronic aortic regurgitation by two dimensional speckle tracking echocardiography.

Methods: Sixty one asymptomatic patients with chronic aortic regurgitation, with no ischemic heart diseases (by coronary angiography) or conductive heart diseases, no diabetes mellitus, no hypertension, and no other valvular heart diseases (group 1) and fifty age and sex-matched healthy subjects (group 2) were enrolled into the study. Group (1) was further classified into 3 sub-groups according to 4 chosen parameters from the published guidelines of American Society of Echocardiography (ASE) into: Mild AR, Moderate AR, and Severe AR.

All patients and controls underwent echocardiographic examination including conventional echocardiography, tissue Doppler study and Two Dimensional (2-D) Speckle Tracking

off value of > (-19.62) has sensitivity and specificity of $91.3 \%$ and $95.5 \%$ respectively, with Positive Predictive Value (PPV) and Negative Predictive Value ( NPV ) of $87.5 \%$ and $96.9 \%$ respectively, Area under curve (AUC) of 0.981. In all types of AR, GLS had higher NPV than PPV which makes it a powerful screening tool for early detection of subtle LV systolic dysfunction.

Conclusion: Global Longitudinal strain measured by 2-D speckle tracking echocardiography is an excellent tool for early detection of subtle LV systolic dysfunction in asymptomatic patients with chronic AR.

Keywords: Aortic Regurgitation, Global Longitudinal Strain, Ejection Fraction.

1 Correspondent author: Consultant Cardiologist, FRCP, CABM, FICMS/cardio, Iraqi Center of Heart Diseases, Baghdad, Iraq.

2 Higher Diploma in Echocardiography, MBChB, DCH, Ibn Al-Nafees Teaching Hospital, Baghdad, Iraq.
\end{abstract} Echocardiography.

Results: GLS showed the highest sensitivity and specificity in detection of subtle LV systolic dysfunction in moderate AR. In moderate AR,a cut
Received at 13/6/2017

Accepted at 4/12/2017

\section{INTRODUCTION}

$\mathrm{A}$ ortic regurgitation (AR) is a common form of valvular heart disease ${ }^{(1)}$ Natural history studies have shown that this results in left ventricular (LV) dilation and, ultimately, systolic dysfunction, which is transiently reversible but then becomes permanent ${ }^{(2-3)}$.

\section{Quantitation of Aortic Regurgitation}

I-Doppler Parameters: ${ }^{(4,5,6,7,8)}$

\begin{tabular}{|c|c|c|c|}
\hline Aortic Regurgitation & Mild & Moderate & Severe \\
\hline Jet density- CW & Incomplete or faint & Dense & Dense \\
\hline Jet deceleration rate CW (PHT, msec) & Slow $>500$ & Medium 500-200 & Steep<200 \\
\hline $\begin{array}{c}\text { Diastolic flow reversal in descending } \\
\text { orta PW }\end{array}$ & $\begin{array}{c}\text { Brief ,early } \\
\text { diastolic reversal }\end{array}$ & Intermediate & $\begin{array}{c}\text { Prominent } \\
\text { holodiastolic reversal }\end{array}$ \\
\hline
\end{tabular}


II- Quantitative Parameters ${ }^{(4,9,10,11,12,13,14,15)}$

\begin{tabular}{|l|c|c|c|}
\hline VC width, cm & $<0.3$ & $0.3-0.60$ & $>0.6$ \\
\hline Jet width/LVOT width, \% & $<25$ & $25-45 \quad 46-64$ & $\geq 65$ \\
\hline Jet CSA/LVOT CSA, \% & $<5$ & $5-20 \quad 21-59$ & $\geq 60$ \\
\hline RVol, mL/beat & $<30$ & $30-44 \quad 45-59$ & $\geq 60$ \\
\hline RF, \% & $<30$ & $30-3940-49$ & $\geq 50$ \\
\hline EROA, $\mathbf{c m}^{2}$ & $<0.10$ & $0.10-0.190 .20-0.29$ & $\geq 0.30$ \\
\hline
\end{tabular}

* AR, Aortic regurgitation; $C S A$, cross sectional area; $C W$, continuous wave Doppler; EROA, effective regurgitant orifice area; $L V$, left ventricle; $L V O T$, left ventricular outflow tract ; msec, millisecond; $P H T$, pressure half-time; $P W$, pulsed wave Doppler; $R$ Vol, regurgitant volume; $R F$, regurgitant fraction; $V C$, vena contracta

LV Global Systolic Function:

-Mitral Annulus Plane Systolic Excursion (MAPSE) and Average myocardial tissue velocity in systole (s): M-mode interrogation is undertaken of the lateral mitral annulus, and annular excursion toward the transducer is then calculated. There is a relatively linear correlation between the magnitude of systolic annular excursion and global systolic function. This same principle is used in Doppler tissue imaging of the annulus for determination of systolic excursion as a marker of ventricular function to measure average systolic myocardial tissue velocity $\left(\mathrm{s}^{\prime}\right) .^{(16,17)}$

- Ejection Fraction:

$\mathrm{EF}$ is calculated from EDV and ESV estimates, using the following formula: $\mathrm{EF}=(\mathrm{EDV}$ ESV)/EDV. Where EDV is the end diastolic volume, ESV is the end systolic volume. The biplane method of disks (modified Simpson's rule) is the currently recommended $2 \mathrm{D}$ method to assess LV EF . . $^{(18,19)}$

- Global Longitudinal Strain (GLS): Lagrangian strain is defined as the change in length of an object within a certain direction relative to its baseline length: ${ }^{20}$ Strain $(\%)=\left(\right.$ L1-L0)/L0 $\quad{ }^{21}$ where $\mathrm{L} 1$ is the length at time t, and L0 is the initial length at time 0 . The most commonly used strain-based measure of LV global systolic function is GLS. It is usually assessed by speckle-tracking echocardiography (STE) ${ }^{(22,23,}$ 24, 25)

The negative nature of GLS can lead to confusion when describing increases or decreases in strain. It is recommended that all references to strain changes specifically mention an increase or decrease in the absolute value of strain, to avoid confusion. ${ }^{23}$
METHOD

A Cross Sectional Study was done in the period from $7^{\text {th }}$ May 2016 to $30^{\text {th }}$ April 2017 in the Department of Echocardiography in AlYarmook Teaching Hospital, Iraqi Center of Heart Diseases, and Baghdad Teaching Hospital.

The study population consisted of 111 individuals, 61 patients with isolated AR referred as (group 1); and 50 healthy persons (control group) referred as (group 2).

- Inclusion criteria were: Asymptomatic patient with chronic aortic regurgitation, with NYHA class I (New York Heart Association Classification of Heart Failure).

- Exclusion criteria were:

1-Ischemic heart disease (excluded by coronary angiography).

2- Arrhythmia and conduction defects.

3- Hypertension.

4- Diabetes mellitus.

5- Other valvular heart diseases.

6- Previous cardiac surgery.

7-Any other chronic systemic diseases.

All 61 patients (group 2) were further divided into three groups (Mild, Moderate and Severe AR); according to the following chosen echocardiographic parameters depending on the published guidelines of ASE ${ }^{(12)}$

1- Vena contracta width.

2- Regurgitant volume.

3- Pressure-half time.

4- Jet width/LVOT width.

The control group consists of 50 healthy volunteers, (men, and women) from the same area who were recruited as control subjects. All control subjects identified themselves as healthy volunteers.

Conventional echocardiography, tissue Doppler and speckle tracking echocardiography were performed to all patients and controls using 
Echocardiography machine; Vivid E9 (GE, Healthcare).

Transthoracic echocardiographic images were acquired at rest with the patient in the left lateral decubitus position by the same echocardiographer. The conventional echocardiography images were taken in parasternal and apical views and the standard LV measurement included: Left Ventricular End Diastolic Diameter (LVEDD), Left Ventricular End Systolic Diameter (LVESD), interventricular septum thickness (IVST) and posterior wall thickness (PWT) using 2D and Mmode measurement. EF\%, EDV, ESV were measured using Simpson's method. MAPSE was measured by $\mathrm{M}$-mode interrogation of the lateral mitral annulus, and annular excursion toward the transducer is then calculated. LV mass was derived from the Devereux-modified cube formula: $\mathrm{LV}$ Mass $=0.8 \quad \boldsymbol{0}$ 1.04. $\left[(\text { IVS+LVID+PWT })^{3}\right.$-LVID $\left.^{\mathbf{3}}\right] \mathbf{+ 0 . 6 g}$

Where IVS is interventricular septum; LVID is $\mathrm{LV}$ internal diameter, and PWT is inferolateral wall thickness.

To assess AR severity, transmitral and aortic flows were recorded using pulsed Doppler echocardiography. Vena contracta width (VCW) was measured using color Doppler in the parasternal long axis view. Continuous wave Doppler through aortic valve in the five chamber view was used to measure PHT.Jet width /LVOT width was measured using color Doppler in the parasternal long axis view. Average septal and lateral (s') was taken using tissue Doppler image through septal and lateral wall of LV to measure average myocardial tissue velocity. The frame rate was adjusted at 80 frames/s, and the cine-loop of 3 consecutive beats was stored for offline speckle-tracking analysis. Images were acquired at end-expiration. Apical 3-chamber, 4chamber and 2-chamber views were used for longitudinal strain Analysis.

In each echocardiographic view, the myocardium was automatically divided into 6 segments. The global longitudinal strain was calculated by averaging the peak systolic values of all the 18 segments, derived from the three apical views (six segments in each apical view): the septal, anteroseptal, lateral, anterior, posterior and inferior LV walls.

SPSS 20. software package was used to make the statistical analysis, $\mathrm{p}$ value was considered when appropriate to be significant if less than 0.05 .

\section{RESULT}

Apart from SBP, DBP and HR, there was no statistically significant difference in age, sex, weight, and height between AR patients and control (Table 1). Table -2- shows conventional echocardiographic parameters in AR according to severity as compared to control .Except for EF\% (in which EF decreased significantly in severe AR), the value of the rest of the variables in table 3 increase as the severity of AR increase.

Table 1: Demographic and clinical data of the study population $(n=111)$

\begin{tabular}{|c|c|c|c|c|c|}
\hline 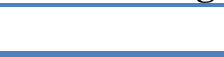 & Normal & Mild & Moderate & Severe & $P$ value \\
\hline Number & 50 & 16 & 23 & 22 & - \\
\hline Age (years) & $47.8 \pm 14.7$ & $51.2 \pm 10.5$ & $50.0 \pm 16.3$ & $48.2 \pm 14.0$ & 0.832 \\
\hline \multicolumn{5}{|c|}{ Sex } & \multirow{3}{*}{0.825} \\
\hline Male (53) & $24(48.0 \%)$ & $6(37.5 \%)$ & $12(52.2 \%)$ & $11(50.0 \%)$ & \\
\hline Female (58) & $26(52.0 \%)$ & $10(62.5 \%)$ & $11(47.8 \%)$ & $11(50.0 \%)$ & \\
\hline Weight (kg) & $74.9 \pm 7.6$ & $69.6 \pm 4.1$ & $74.3 \pm 6.9$ & $73.7 \pm 6.4$ & 0.068 \\
\hline Height $(\mathrm{cm})$ & $172.8 \pm 5.7$ & $172.8 \pm 8.2$ & $175.0 \pm 6.6$ & $172.7 \pm 6.3$ & 0.509 \\
\hline SBP & $128.6 \pm 5.7$ & $129.7 \pm 5.3$ & $132.1 \pm 4.6^{*}$ & $132.6 \pm 5.0^{*}$ & 0.009 \\
\hline DBP & $73.3 \pm 6.1$ & $72.9 \pm 6.7$ & $73.3 \pm 7.2$ & $80.5 \pm 7.0^{*}$ & $<0.001$ \\
\hline HR & $62.4 \pm 5.1$ & $62.3 \pm 4.5$ & $65.8 \pm 5.1$ & $68.0 \pm 5.1^{*}$ & 0.003 \\
\hline
\end{tabular}

DBP, Diastolic Blood Pressure; HR, Heart Rate; SBP, Systolic Blood Pressure 
Table 2: echocardiographic data of the study population $(n=111)$

\begin{tabular}{|c|c|c|c|c|c|}
\hline & Normal & Mild & Moderate & Severe & $P$ value \\
\hline Number & 50 & 16 & 23 & 22 & - \\
\hline IVS thickness & $0.77 \pm 0.10^{\mathrm{a}}$ & $0.82 \pm 0.09^{\mathrm{a}}$ & $0.82 \pm 0.08^{\mathrm{a}}$ & $0.97 \pm 0.15^{* * * b}$ & $<0.001$ \\
\hline Posterior wall thickness & $0.81 \pm 0.10^{\mathrm{a}}$ & $0.87 \pm 0.09^{\mathrm{a}}$ & $1.02 \pm 0.07^{* * * * b}$ & $1.12 \pm 0.10^{* * * * \mathrm{c}}$ & $<0.001$ \\
\hline LV mass & $128.8 \pm 30.3^{\mathrm{a}}$ & $129.7 \pm 23.1^{\mathrm{a}}$ & $184.1 \pm 29.4^{* * * b}$ & $284.7 \pm 62.5^{* * *_{c}}$ & $<0.001$ \\
\hline LVEDD & $4.85 \pm 0.49^{\mathrm{a}}$ & $4.65 \pm 0.28^{\mathrm{a}}$ & $5.36 \pm 0.35^{* * * * b}$ & $6.25 \pm 0.55^{* * * *} \mathrm{c}$ & $<0.001$ \\
\hline LVESD & $2.82 \pm 0.48^{\mathrm{a}}$ & $3.03 \pm 0.22^{\mathrm{a}}$ & $3.89 \pm 0.36^{* * * b}$ & $4.45 \pm 0.52^{* * * c}$ & $<0.001$ \\
\hline LVED volume & $103.6 \pm 16.2^{a}$ & $110.4 \pm 10.5^{\mathrm{a}}$ & $142.2 \pm 25.8^{* * * b}$ & $234.8 \pm 58.3^{* * * * c}$ & $\overline{<0.001}$ \\
\hline LVES volume & $34.9 \pm 7.7^{\mathrm{a}}$ & $39.3 \pm 5.1^{\mathrm{a}}$ & $46.8 \pm 8.4^{\mathrm{a}}$ & $51.1 \pm 28.5^{* * * b}$ & $<0.001$ \\
\hline$\overline{\text { EF\% }}$ & $66.5 \pm 4.1$ & $64.4 \pm 3.5$ & $67.0 \pm 2.01$ & $57.3 \pm 4.4^{* * * *}$ & $\overline{<0.001}$ \\
\hline$\overline{\text { LA Dimension }}$ & $\overline{35.7 \pm 2.0}$ & $\overline{36.0 \pm 2.5}$ & $38.3 \pm 3.3^{* * * *}$ & $42.8 \pm 4.0^{* * * *}$ & $\overline{<0.001}$ \\
\hline$\overline{\text { Ascending Aorta D }}$ & $2.93 \pm 0.30$ & $2.82 \pm 0.21$ & $3.00 \pm 0.25$ & $4.37 \pm 0.49^{* * *}$ & $\leq 0.001$ \\
\hline
\end{tabular}

Table (3) displays the systolic parameters used in control and AR patients as follows: Absolute GLS value showed statistically significant decrease in moderate and severe $\mathrm{AR}$; $\mathrm{P}$ value $<0.001$. However; s`and EF \% showed only a statistically significant decrease in severe AR versus control. Table (4) presents the linear regression analysis of different variables versus GLS according to severity of AR. In severe AR, EF and MAPSE showed significant negative relationship ( $\beta$ ) with numerical value of GLS (that is reflected as positive relationship with absolute GLS value) with a $\mathrm{P}$ value of $<0.001$.

Table 3: Change in Global Longitudinal Strain values as compared to other systolic echocardiographic parameters of the study population $(n=111)$

\begin{tabular}{|l|c|c|c|c|c|}
\hline & Normal & Mild & Moderate & Severe & $\begin{array}{c}\text { P } \\
\text { value }\end{array}$ \\
\hline Number & 50 & 16 & 23 & 22 & - \\
\hline Average mitral annular s' & $11.62 \pm 1.42$ & $11.64 \pm 1.40$ & $10.59 \pm 1.03$ & $6.91 \pm 0.90^{*}$ & $<0.001$ \\
\hline Global longitudinal Strain \% & $-22.42 \pm 1.88$ & $-20.88 \pm 1.39$ & $-7.73 \pm 1.27$ & $-15.07 \pm 1.96^{* *}$ & $<0.001$ \\
\hline Average MAPSE & $1.27 \pm 0.13$ & $1.28 \pm 0.07$ & $1.28 \pm 0.08$ & $1.24 \pm 0.15$ & 0.534 \\
\hline EF\% & $66.5 \pm 4.1$ & $64.4 \pm 3.5$ & $67.0 \pm 2.01$ & $57.3 \pm 4.4^{* * *}$ & $<0.001$ \\
\hline
\end{tabular}

Table 4: Linear regression analysis of different variables versus GLS in different types of AR:

\begin{tabular}{|l|c|c|c|c|c|c|}
\hline \multicolumn{1}{|c|}{ Variables } & \multicolumn{2}{c|}{ Mild AR } & \multicolumn{2}{c|}{ Moderate AR } & \multicolumn{2}{c|}{ Severe AR } \\
\hline IVS thickness & $\mathrm{B}$ & $\mathrm{P}$ value & $\mathrm{B}$ & $\mathrm{P}$ value & $\mathrm{B}$ & P value \\
\hline Post. wall thickness & 0.010 & 0.485 & 0.054 & 0.403 & 0.029 & 0.448 \\
\hline LV mass & 0.160 & 0.278 & 0.134 & 0.271 & 0.146 & 0.258 \\
\hline LVEDD & 0.145 & 0.181 & 0.121 & 0.067 & 0.130 & 0.152 \\
\hline LVESD & 0.197 & 0.132 & 0.125 & 0.569 & 0.983 & $<0.001[$ S.] \\
\hline LVED volume & 0.211 & 0.057 & 0.077 & 0.727 & 0.985 & $<0.001[\mathrm{~S}]$. \\
\hline LVES volume & 0.150 & 0.175 & 0.932 & $<0.001[\mathrm{~S}]$. & 0.982 & $<0.001[\mathrm{~S}]$. \\
\hline EF\% & 0.006 & 0.492 & 0.140 & 0.135 & 0.967 & $<0.001[\mathrm{~S}]$. \\
\hline Average MAPSE & -0.106 & 0.125 & -0.153 & 0.243 & -0.869 & $<0.001[\mathrm{~S}]$. \\
\hline LA Dimension & -0.186 & 0.245 & -0.222 & 0.310 & -0.972 & $<0.001[\mathrm{~S}]$. \\
\hline Ascending Aorta D & 0.150 & 0.289 & 0.121 & 0.067 & 0.971 & $<0.001[\mathrm{~S}]$. \\
\hline
\end{tabular}

IVS , interventricular septum ; LVEDD, left ventricular diastolic diameter; LVESD, left ventricular systolic diameter 
Figure (1) reveals this relation according to severity of AR. EF showed statistically significant relationship in severe AR (but not in mild and moderate AR), which means that it was unable to detect early LV systolic dysfunction as compared to GLS. Table (5) explains the predictive characteristics of systolic parameters used for detection of early Left ventricular systolic dysfunction in asymptomatic AR patient From the AR study sample, a GLS cut off value of >- $19.62 \%$ yielded a sensitivity and specificity of $75.4 \%$ and $99 \%$ respectively, both of which are higher as compared to EF and s' .The area under curve (AUC) being 0.928, which reflected an excellent test.
This means that it was the most diagnostic and better confirmatory and screening tools for early detection of subtle reduction in LV systolic function. Finally; table (6) and figure (2) analyses the predictive characteristics of GLS for different types of AR according to severity. In the recent study, a GLS cut off value of > $19.62 \%$ for moderate AR showed sensitivity and specificity of $91.3 \%, 95.5 \%$ for moderate AR with an AUC of 0.981 (excellent test) .This cut off value showed the best positive and negative predictive values being $87.5 \%$ and $96.9 \%$ respectively. This means that GLS was both confirmatory and exclusive tool in the early diagnosis of LV systolic dysfunction.
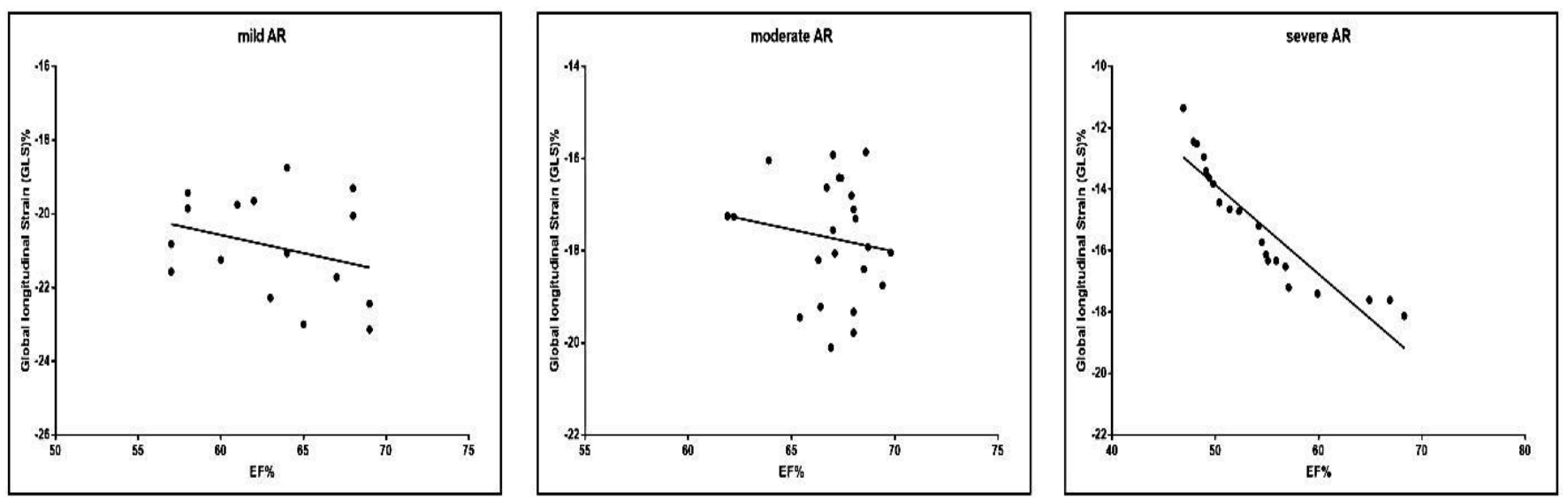

Figure 1: Relationship between EF \% versus GLS in patients according to severity of AR.

Table 5: Predictive characteristics of echocardiographic variables for the detection of left ventricular systolic dysfunction in asymptomatic patients with chronic aortic regurgitation

\begin{tabular}{|l|l|l|l|l|l|}
\hline & Cut off & Sensitivity & Specificity & AUC & P value \\
\hline GLS\% & $>-19.62$ & $75.4 \%$ & $99 \%$ & 0.928 & $<0.0001$ \\
\hline Average mitral annular s' & $\leq 9.65$ & 57.4 & $94 \%$ & 0.815 & $<0.0001$ \\
\hline EF & $\leq 57.1$ & $31.1 \%$ & $98.0 \%$ & 0.680 & $<0.0001$ \\
\hline MAPSE & - & - & - & 0.519 & 0.729 \\
\hline
\end{tabular}

Table 6: Predictive characteristics of GLS for the detection of left ventricular systolic dysfunction in asymptomatic patients with chronic aortic regurgitation (According to severity)

\begin{tabular}{|l|l|l|l|l|l|l|l|}
\hline & Cut off & Sensitivity & Specificity & PPV & NPV & AUC & P value \\
\hline Mild AR & $>-22.54$ & $87.5 \%$ & $54.0 \%$ & $37.8 \%$ & $93.1 \%$ & 0.739 & 0.0004 \\
\hline Moderate AR & $>-19.62$ & $91.3 \%$ & $95.5 \%$ & $87.5 \%$ & $96.9 \%$ & 0.981 & $<0.0001$ \\
\hline Severe AR & $>-18.2$ & $100 \%$ & $83.2 \%$ & $84.5 \%$ & $100 \%$ & 0.963 & $<0.0001$ \\
\hline
\end{tabular}

AUC, area under curve; $N P V$, negative predictive value; PPV, positive predictive value. 

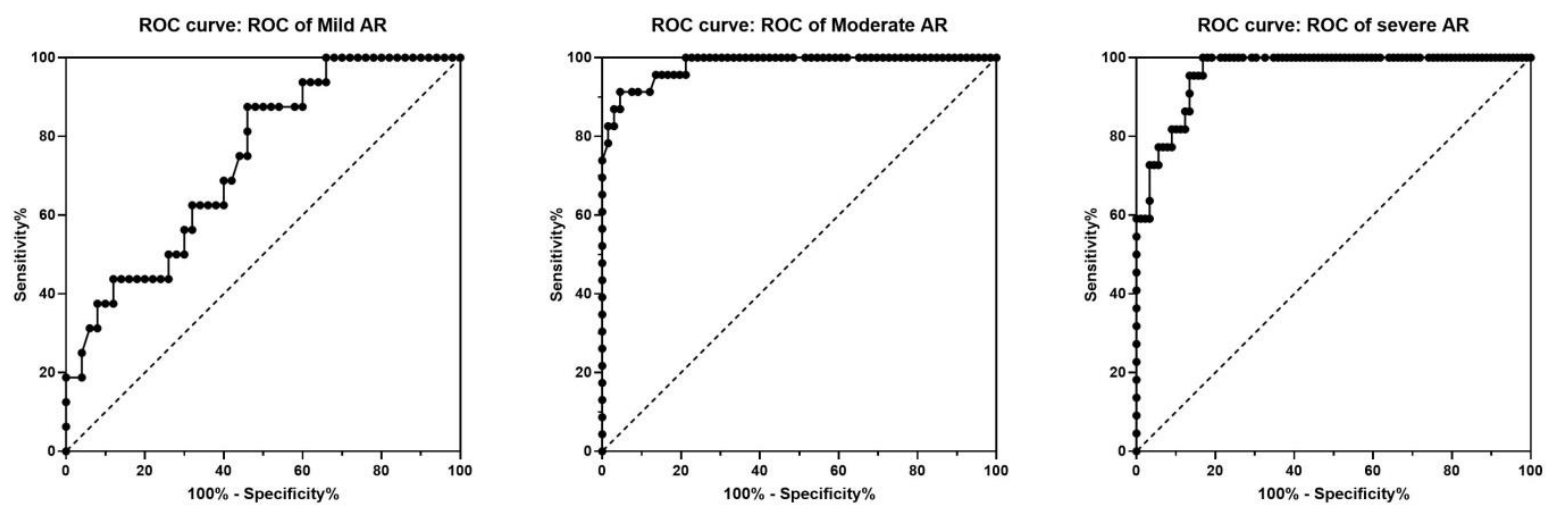

Figure (2): Receiver Operating Characteristic Curve (ROC) curve analysis to predict the GLS validity to discriminate the degree of AR.

\section{DISCUSSION}

The current study showed that apart from SBP, DBP and HR; there was no significant statistic relationship of age, sex, body weight or height with severity of AR.

In a study reported by Vaida Mizarienè et al (study 2012) 26 ,it was only systolic blood pressure which showed statistically significant change with severe AR, which is close to the results of this study.

Absolute Global longitudinal strain value showed statistically significant reduction in moderate and severe AR. Although average MAPSE was reduced with increase in severity of AR, that change was not statistically significant. $\mathrm{EF}$ and average mitral annular s' were reduced with increasing severity of AR. However; measurement ensued significant results only in severe AR.

In their study, Marciniak et al ${ }^{27}$ reported that only severe AR that showed significant change in EF. In their study, they did not measure global longitudinal strain, instead; they measured peak systolic strain for interventricular septum and LV lateral wall .Their study showed a significant reduction in absolute peak systolic strain value in interventricular septum and LV lateral wall in moderate and severe AR. These findings are close to the finding in the recent study. Both EF and MAPSE have statistically significant negative relationship with numerical value of GLS in severe AR.

Vaida Mizarienè et $\mathrm{al}^{24}$, studied this relationship for EF with GLS in patients with severe AR.
The results were concordant to the recent study with a $P$ value of 0.001 .

The recent study focused on sensitivity and specificity of the GLS and compared them with that of EF, average mitral annular s' and MAPSE. In all types of AR, GLS has more sensitivity and specificity to detect early LV systolic dysfunction. The study also calculated the positive predictive value (PPV) and negative predictive value (NPV) for each type of AR. Moderate AR showed PPV and NPV of $87.5 \%$ and $96.9 \%$ respectively. In severe AR, the PPV and NPV of $84.5 \%$ and $100 \%$ respectively.

In 2015, See Hooi Ewe et el ${ }^{28}$, studied left ventricular systolic dysfunction in asymptomatic patient with AR as a whole without grading severity .This was a retrospective study which used only apical 4 chamber view to measure the GLS. GLS cut off value of $-15.1 \%$ has a sensitivity of $35 \%$ and specificity of $87 \%$ ( PPV of $75 \%$ and NPV of $54 \%$ ). The latter reduced sensitivity may be attributed to the use of single view to measure GLS. This means that other segments which should be measured in Apical three and two chamber views are missed and thus might lead to this lower sensitivity.

\section{Limitations of the study:}

Other types of strain such as radial and circumferential strain were not measured because of unavailability of the software in the used echocardiographic machine. The values of latter types of strain may be more informative. 


\section{CONCLUSION}

The study showed that GLS was the most sensitive and specific to detect early systolic dysfunction in chronic AR. Thus it can be a gold standard for assessing subtle LV systolic dysfunction in asymptomatic patients with chronic AR and preserved EF.

The study concluded that all parameters have negative relationship with absolute value of GLS, except for EF and MAPSE which have positive relation.

\section{REFRENCES}

1. Supino PG, Borer JS: Epidemiology of valvular heart diseases: a growing public health problem, Heart Failure Clinic. 2006 Oct; 2(4):379-93.

2. Nicole M. Bhave, MD : Aortic Regurgitation:Etiology, Left Ventricular Response and Role of Echocardiography , Roberto $\mathrm{M}$ et al ,Ase's Comprehensive Echocardiography, $2^{\text {nd }}$ edition, Philadelphia, 2016 ,Section XVI ,Chapter 103 ,p 438 .

3. Catherine M. Otto and Robert O. Bonow: Aortic Regurgitation: Cause and Pathology, Douglas L. Mann, MD et al., Braunwald E. Heart Disease: A Textbook of Cardiovascular Medicine, 10th ed. Philadelphia, Pa: Elsevier Saunders; 2015,Chapter $63, \mathrm{p} 1458$.

4. William A. Zoghbi et al : Recommendations for Evaluation of the Severity of Native Valvular Regurgitation with Two-dimensional and Doppler Echocardiography: A report from the American Society of Echocardiography's Nomenclature and Standards Committee and The Task Force on Valvular Regurgitation, developed in conjunction with the American College of Cardiology Echocardiography Committee, The Cardiac Imaging Committee Council on Clinical Cardiology, the American Heart Association, and the European Society of Cardiology Working Group on Echocardiography, July 2003,p789.

5. Grayburn PA, Smith MD, Handshoe R, Friedman BJ, DeMaria AN:Detection of aortic insufficiency by standard echocardiography, pulsed Doppler echocardiography, and auscultation: a comparison of accuracies. Ann Intern Med 1986;104:599-605.

6. Perry GJ, Helmcke F, Nanda NC, Byard C, Soto B: Evaluation of aortic insufficiency by Doppler color flow mapping.J Am Coll Cardiol 1987;9:952-9.

7. Enriquez-Sarano M, Bailey KR, Seward JB, Tajik AJ, Krohn MJ, Mays JM. Quantitative Doppler assessment of valvular regurgitation. Circulation 1993;87:841-8.

8. Touche T, Prasquier R, Nitenberg A, de Zuttere D, Gourgon R. Assessment and follow-up of patients with aortic regurgitation by an updated Doppler echocardiographic measurement of the regurgitant fraction in the aortic arch. Circulation 1985; 72:819-24.

9. Braverman AC, Thompson RW, Sanchez LA: Diseases of the aorta. In Bonow R,editor: Braunwald's heart disease, 9 ed, Philadelphia, 2011, WB Saunders, pp 1309-1337.

10. Bekeredjian, Raffi; Grayburn, Paul A.: "Valvular Heart Disease Aortic Regurgitation". Circulation,2005,112 (1): 125-134.

11. Tornos P, Evangelista A, Bonow RO: Aortic regurgitation. In Otto $\mathrm{CM}$, Bonow $\mathrm{RO}$ (eds): Valvular Heart Disease: A Companion to Braunwald's Heart Disease. 4th ed. Philadelphia,Saunders, 2013, pp 163-178

12. Babu AN, Kymes SM, Carpenter Fryer SM. Eponyms and the diagnosis of aortic regurgitation: what says the evidence?. Ann Intern Med. 2003 May 6. 138(9):736-42.

13. Reynolds T, Abate J, Tenney A, Warner MG: The $\mathrm{JH} / \mathrm{LVOH}$ method in the quantification of aortic regurgitation: how the cardiac sonographer may avoid an important potential pitfall.J Am Soc Echocardiogr 1991;4:105-8.

14. Tribouilloy CM, Enriquez-Sarano M, Bailey KR, Seward JB,Tajik AJ. Assessment of severity of aortic regurgitation using the width of the vena contracta: a clinical color Doppler imaging study. Circulation 2000;102:558-64.

15. Enriquez-Sarano M, Seward JB, Bailey KR, Tajik AJ. Effective regurgitant orifice area: a noninvasive Doppler development of an old hemodynamic concept. J Am Coll Cardiol 1994;23:443-51.

16. Armstrong, William F.; Ryan, Thomas: Aortic Regurgitation ,Feigenbaum's Echocardiography, 7th Edition, Copyright ,2010 ,Chapter 11, p 544.

17. Stuart J Hutchison: Aortic Insufficiency, Principles of Echocardiography and Intracardiac Echocardiography, Elsevier Saunders,2012,P 42.

18. Roberto M. Lang, MD :Recommendations for Cardiac Chamber Quantification by Echocardiography in Adults: An Update from the American Society of Echocardiography and the European Association of Cardiovascular Imaging. Jan 2015.p 6.

19. Dorosz JL, Lezotte DC, Weitzenkamp DA, Allen LA, Salcedo EE. Performance of 3-dimensional echocardiography in measuring left ventricular volumes and ejection fraction: a systematic review and meta-analysis. J Am Coll Cardiol 2012;59:1799-808.

20. Reisner SA, Lysyansky P, Agmon Y, Mutlak D, Lessick J, Friedman Z.Global longitudinal strain: 
a novel index of left ventricular systolic function.

J Am Soc Echocardiogr 2004;17:630-3.

21. Dalen H, Thorstensen A, Aase SA, Ingul CB, Torp H, Vatten LJ, et al.Segmental and global longitudinal strain and strain rate based on echocardiography of 1266 healthy individuals: the HUNT study in Norway.Eur $J$ Echocardiogr 2010;11:176-83.

22. Voigt JU, Pedrizzetti G, Lysyansky P, Marwick TH, Houle HC,Baumann R, et al. Definitions for a Common Standard for 2D Speckle Tracking Echocardiography. Consensus document of the EACVI/ASE/Industry Task Force to Standardize Deformation Imaging. Eur Heart J Cardiovasc Imaging 2014 (in press).p 1-11.

23. Mor-Avi V, Lang RM, Badano LP, Belohlavek M, Cardim NM,Derumeaux G, et al. Current and evolving echocardiographic techniques for the quantitative evaluation of cardiac mechanics: ASE/EAE consensus statement on methodology and indications endorsed by the Japanese Society of Echocardiography. J Am Soc Echocardiogr 2011;24:277-313.

24. Kocabay G, Muraru D, Peluso D, Cucchini U, Mihaila S, Padayattil-Jose S,et al. Normal left ventricular mechanics by two-dimensional speckle tracking echocardiography. Revista Espanola de Cardiologia 2014;67:651-8.
25. Kuznetsova T, Herbots L, Richart T, D’Hooge J, Thijs L, Fagard RH, et al.Left ventricular strain and strain rate in a general population. Eur Heart $\mathrm{J}$ 2008;29:2014-23.

26. Vaida Mizarienė1, 2, Silvija Bučytė1, Diana Žaliaduonytè-Pekšienè2, Regina Jonkaitienė2,Jūratė Janėnaitė1, Jolanta Vaškelytė et al: Components of Left Ventricular Ejection and Filling in Patients with aortic Regurgitation assessed by speckle-tracking echocardiography, Medicina (Kaunas) 2012;48(1):31-8.

27. Anna Marciniak et al: Myocardial deformation abnormalities in patients with aortic regurgitation: a strain rate imaging study, European Journal of Echocardiography (2009) 10, 112-119 .

28. See Hooi Ewe, Marlieke L.A. Haeck1, Arnold C.T. Ng, Tomasz G. Witkowski,Dominique Auger, Darryl P. Leong et al : Detection of subtle left ventricular systolic dysfunction in patients with significant aortic regurgitation and preserved left ventricular ejection fraction: speckle tracking echocardiographic analysis, European Heart Journal - Cardiovascular Imaging (2015) 16, 992-999. 